\title{
Nanoscale
}

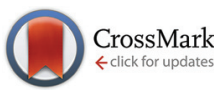

Cite this: Nanoscale, 2016, 8, 13730

\section{Directing lineage specification of human mesenchymal stem cells by decoupling electrical stimulation and physical patterning on unmodified graphene}

\author{
Daniel A. Balikov, $\dagger^{a}$ Brian Fang, $\dagger^{a, b}$ Young Wook Chun, $\uparrow^{a, c}$ Spencer W. Crowder, $\S \dagger^{a}$ \\ Dhiraj Prasai, ${ }^{b}$ Jung Bok Lee, ${ }^{a}$ Kiril I. Bolotin ${ }^{\mathrm{b}}$ and Hak-Joon Sung ${ }^{\star a, c, d}$
}

The organization and composition of the extracellular matrix (ECM) have been shown to impact the propagation of electrical signals in multiple tissue types. To date, many studies with electroactive biomaterial substrates have relied upon passive electrical stimulation of the ionic media to affect cell behavior. However, development of cell culture systems in which stimulation can be directly applied to the material - thereby isolating the signal to the cell-material interface and cell-cell contracts - would provide a more physiologically-relevant paradigm for investigating how electrical cues modulate lineagespecific stem cell differentiation. In the present study, we have employed unmodified, directly-stimulated, (un)patterned graphene as a cell culture substrate to investigate how extrinsic electrical cycling influences the differentiation of naive human mesenchymal stem cells (hMSCs) without the bias of exogenous biochemicals. We first demonstrated that cyclic stimulation does not deteriorate the cell culture media or result in cytotoxic $\mathrm{pH}$, which are critical experiments for correct interpretation of changes in cell behavior. We then measured how the expression of osteogenic and neurogenic lineage-specific markers were altered simply by exposure to electrical stimulation and/or physical patterns. Expression of the early osteogenic transcription factor RUNX2 was increased by electrical stimulation on all graphene substrates, but the mature marker osteopontin was only modulated when stimulation was combined with physical patterns. In contrast, the expression of the neurogenic markers MAP2 and $\beta_{3}$-tubulin were enhanced in all electrical stimulation conditions, and were less responsive to the presence of patterns. These data indicate that specific combinations of non-biological inputs - material type, electrical stimulation, physical patterns - can regulate hMSC lineage specification. This study represents a substantial step in understanding how the interplay of electrophysical stimuli regulate stem cell behavior and helps to clarify the potential for graphene substrates in tissue engineering applications.

Received 31st May 2016, Accepted 28th June 2016 DOI: $10.1039 / c 6 n r 04400$ j www.rsc.org/nanoscale

\section{Introduction}

The nervous system serves as the conduit that carries electrical impulses throughout the body. These signals convey information to and from the brain that range from sensory perception, such as sight, touch and taste, to motor function like the heart beat or muscle contraction. Equally important, this process plays a pivotal role in instructing development and regeneration of body parts. ${ }^{1-3}$ Generally speaking, cells receive and generate paracrine signals through cytokines, growth factors and hormones to communicate with each other, by which their group activities are determined to repair and regenerate damaged cells and tissues. In the case of neuronal impulses in healing, the extracellular matrix (ECM) can help facilitate electrical signal transfer and this process modulates the cell-cell communication in peripheral and central nervous 
system repair..$^{4-8}$ Despite the ECM's important role in transferring electrical signals, most electrical stimulation applied to cells in vitro has relied on electrodes suspended in cell culture media, yielding heterogeneous pulse propagation in the test cell population with formation of a harmful $\mathrm{pH}$ gradient in the culture media. ${ }^{9}$ Furthermore, most studies to-date have used varying parameters to electrically stimulate cells, which impedes the overall progress of the research community by convoluting our interpretation of how specific cellular responses can be achieved with external electrophysical cues. Therefore, methodical development and testing of new tailorable, scalable cell culture systems in which electrical stimulation can be directly applied onto the matrix are currently of great interest to elucidate and clarify the complex biology that responds to extrinsic stimulation.

Graphene, a single atomic monolayer of graphite, has received unprecedented attention over the last decade due to its unique electronic, mechanical, and thermal properties. ${ }^{10,11}$ The exceptional electrical conductivity of graphene has been exploited for a range of applications, but thorough investigation of how stimulation of pristine graphene affects cell behaviour is lacking in the literature. A recent paper by Kim et al. demonstrated that patterned graphene substrates can preferentially encourage osteogenic or neurogenic differentiation when presented as columns or grids, respectively. ${ }^{12}$ However, the cells used in this study were pre-differentiated toward either an osteoblastic or neuronal lineage and the graphene was reduced to graphene oxide, which is known to change the conformation and functionality of adsorbed proteins. In parallel, our group published an early report demonstrating that threedimensional graphene foams encouraged the spontaneous differentiation of hMSCs toward an osteogenic lineage; the materials used in our study were coated with gelatin to enhance cell attachment which could have affected the intrinsic cues of the graphene substrate. Therefore, a logical and necessary next step for the field as a whole would be to employ pristine, unmodified graphene and naïve, unbiased stem cells to more clearly elucidate how patterns affect lineage commitment, and to add cyclical electrical stimulation to mimic the physiological signals received by cells in electroactive tissues.

In this study, we aimed to decouple the effects of (1) material type, (2) application of external, cyclical stimulation, and (3) presentation of physical patterns in regulating the behaviour of naïve, bone marrow-derived hMSCs over a three-day period. By presenting unmodified, uncoated graphene to the cells in the absence of induction media, the impact of each parameter could be clearly elucidated. Following previous findings, we used graphene that was either unpatterned (i.e. flat, uniform) or prepared in columnar or grid-like patterns. We first demonstrated that cyclic electrical stimulation did not change local $\mathrm{pH}$ on the graphene culture substrates, confirming that the culture system was not cytotoxic. We then systematically measured the combined effects of electrical stimulation and physical patterns, and determined a divergent lineage commitment response: expression of the early osteogenic tran- scription factor RUNX2 is enhanced by electrical stimulation on all substrates tested, but the late osteogenic marker, osteopontin, was enhanced only on patterned substrates with concurrent stimulation. Expression of the neurogenic markers $\beta_{3^{-}}$ tubulin and MAP2 were enhanced by electrical stimulation, and this effect was less responsive in the presence of patterns. These data indicate that lineage commitment can be affected by all three tested parameters (material type, electrical stimulation, and physical patterning) without biasing the cellular response with predifferentiation or coating with bioactive molecules. This work helps to clarify the ability of graphene to regulate cell behaviour and can be used to design instructive biomaterial scaffolds for tissue engineering and regenerative medicine applications.

\section{Results and discussion}

\section{Generation of the electrically stimulated graphene cell culture system with highly customizable, low cost materials}

In order to enable cyclic electrical stimulation in a cell culture setting, monolayer graphene was patterned and a platinum wire electrode was epoxyed onto the graphene surface (Fig. 1). Monolayer graphene was subjected to photolithography and reactive ion etching (RIE) in order to generate desired surface patterns (Fig. 2A-C). Human MSCs (hMSCs) obtained from bone marrow (Fig. 1A) were cultured on the graphene substrates (Fig. 1B) with pre-patterned surfaces (Fig. 1C). When the cells are adhered to the graphene surface, voltage was applied to this cell culture system (Fig. 1D) using an Arduino UNO op-amp setup (Fig. 1E). The platinum wire electrodes were positioned such that one electrode contacted the graphene near the outer edge of the substrate while the second electrode was suspended in the cell culture media (Fig. 1F). This assembly of components allowed for ease of fabrication, customizable patterning, low cost of materials and the ability to accurately and precisely tune the electrical stimulation parameters applied onto substrates.

Patterns were fabricated as either grids (Fig. 2B) and columns (Fig. 2C), and the dimensions were comparable to those previously reported. ${ }^{12}$ Because the graphene needed to be transferred to a cell culture setting, copper-based chemical vapor deposition $(\mathrm{CVD})^{13}$ was utilized, allowing for postgrowth transferring of monolayer graphene to polydimethylsiloxane (PDMS) (see Methods for details). PDMS is a material that naturally repels proteins and resists cell adhesion, easy to mold, and non-toxic to cells. Hence, it became the optimal support substrate for graphene. Prior to transfer onto PDMS, the graphene was characterized via Raman spectroscopy. As seen in Fig. 2D, the narrow $\mathrm{G}$ peak $\left(1560 \mathrm{~cm}^{-1}\right)$ confirmed pure graphene was synthesized. A widened $G$ peak indicates contamination by graphene oxide formation. ${ }^{14} \mathrm{~A}$ monolayer character of graphene was confirmed by observing the $2 \mathrm{D}$ peak at $2700 \mathrm{~cm}^{-1}$, a feature absent for multilayer graphite. ${ }^{15}$

PDMS was also coated with Pluronic ${ }^{\circledR}$ to further prevent cell attachment ${ }^{16}$ (Fig. 1B). Pluronic ${ }^{\circledR}$ is an amphilic molecule 


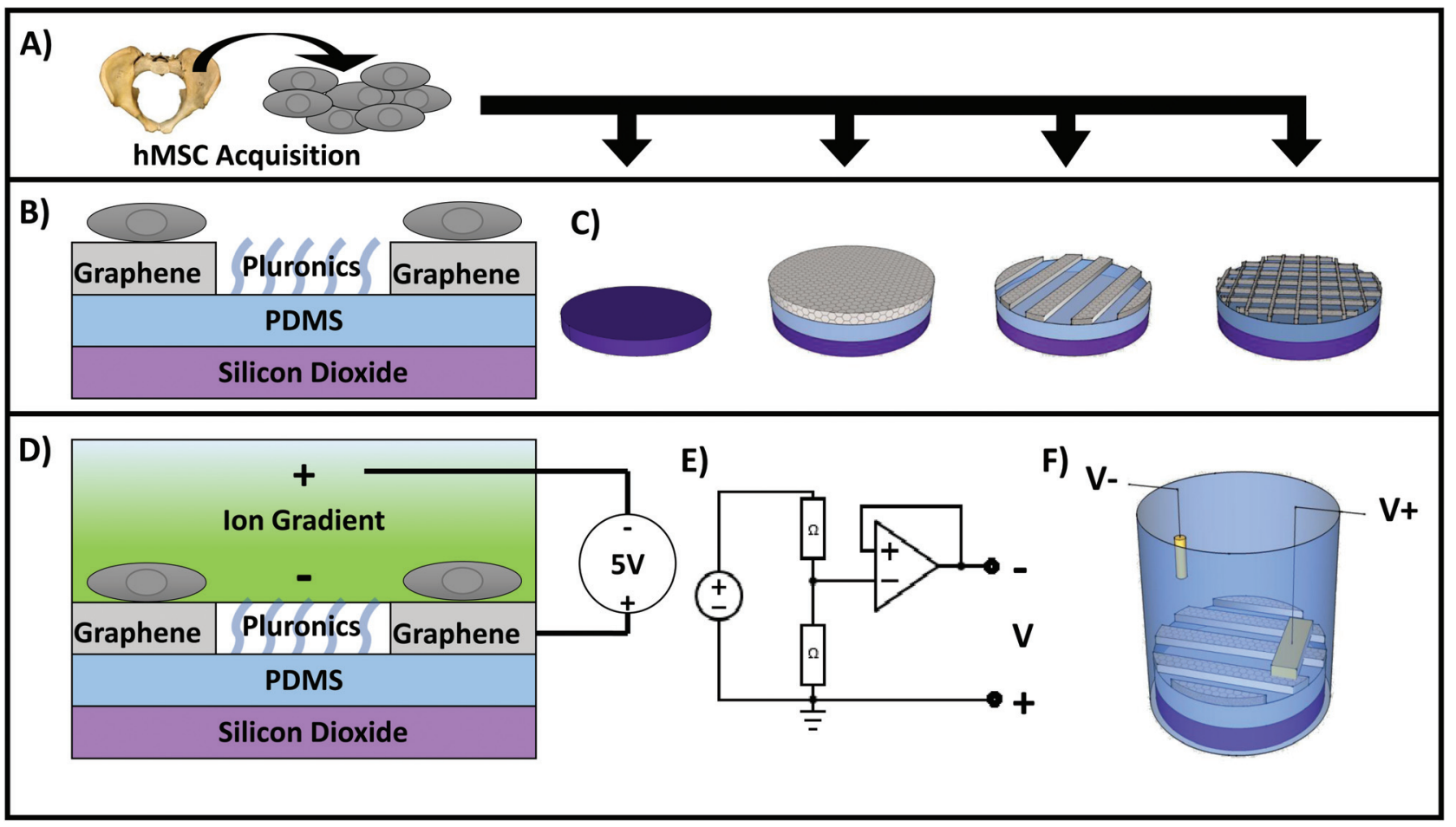

Fig. 1 Experimental setup for graphene substrate experiments. (A) hMSCs acquired from bone marrow are expanded for seeding onto the glass and monolayer graphene substrates. (B) Graphene substrates are backed onto PDMS, which serves as a sturdy frame to transport the graphene substrates. The PDMS was then adhered to silicon dioxide coverglass. Pluronic ${ }^{\circledR}$ was added to the assembled graphene substrates to cover exposed PDMS in order to sequester cell attachment solely with graphene. (C) Cells were seeded on four substrates (from left to right: glass, flat graphene, column graphene, and grid graphene). (D) Once the hMSCs were adhered to the substrates, the circuit was setup (E) with an applied voltage of $5 \mathrm{~V}$. (F) The cathode was epoxyed to the graphene surface while the anode was submerged in the culture media.

and thus hydrophobically bonds the PDMS while its hydrophillic tails prevent protein adsorption and cell adhesion. In this way, hMSCs were forced to only interact with graphene. These final assembled substrates were used for all the in vitro cell experiments.

\section{Optimization of stimulation parameters to prevent cell death}

Next, as a control experiment, cyclic voltammetry of the cell culture media from $-1 \mathrm{~V}$ to $+1 \mathrm{~V}$ for 10 cycles indicated a voltage range that did not break down the culture media (Fig. 2E). The voltage test-range was performed as prior studies confirmed the voltage ceiling was permissible for normal hMSC physiological activity. ${ }^{17,18}$ Between 0 and $0.5 \mathrm{~V}$ the current remained close to $0 \mu \mathrm{A}$ before exponentially increasing above $10 \mu \mathrm{A}$ at higher voltages. We did not exceed $0.5 \mathrm{~V}$ when measuring the media $\mathrm{pH}$ under electrical stimulation because current densities beyond $10 \mu \mathrm{A}$ affects electron transport within proteins and alters their structure, as has been reported for gold electrodes ${ }^{19}$ thus rendering such proteins unnatural in the cell environment. Additionally, break down of the media can cause local $\mathrm{pH}$ change that in turn can influence cell behavior negatively. As seen in Fig. 2F, a significant decrease in $\mathrm{pH}$ was seen at $500 \mathrm{mV}$ across all three tested frequencies after 24 hours of constant stimulation. Therefore, a ceiling voltage of $0.3 \mathrm{~V}$ was chosen to prevent irreversible electrochemical reactions that affect the cell media or cell membrane proteins. This choice is corroborated by previous research reporting that $250 \mathrm{mV}$ stimulus promoted neurogenesis in hMSCs as well as another reporting both TUJ1 ( $\beta_{3}$-tubulin) and MAP2 expression were significantly increased after 1000 seconds of constant electrical stimulation at a similar voltage. $^{20}$

Having characterized the response of the cell culture setup without cells, hMSC were cultured under electrical stimulation on glass and unpatterned graphene. A live/dead assay on electrically stimulated hMSCs was performed to further optimize the stimulation parameters (Fig. 3). $1 \mathrm{~Hz}$ stimulation frequency was chosen because this frequency has been shown to promote neural stem cell health and development in several gold-standard in vitro models. ${ }^{21,22}$ Studies using stimulation frequencies that exceeded $10 \mathrm{~Hz}$ revealed that bone marrowderived hMSCs became chondrogenic and osteogenic, although these experiments were not performed on graphene. ${ }^{23}$ Across all voltages, more cells attached to the glass surface compared to the graphene surface. Despite the observed decreased cell attachment, the elongated spindle morphology of the hMSCs was enhanced on the graphene surfaces compared to glass. Both the decreased number of attached cells and the more pronounced spindle morphology 


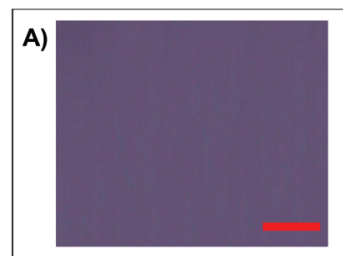

D)



B)



C)

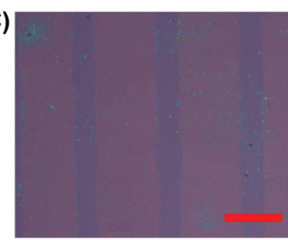

E)

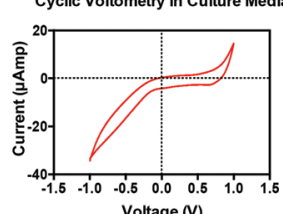

F)



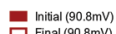

Fig. 2 Substrate characterization and electrical stimulation validation with culture media. (A-C) Unpatterned and patterned graphene deposited on copper foil was imaged to verify the homogeneity of the desired surface patterning. Scale bar $=50 \mu \mathrm{m}$ (D) Raman spectroscopy validated that the graphene generated was monolayer graphene and pure of any chemical contaminants. (E) When cyclic voltammetry was applied to the cell culture media, the boundary voltages that could be applied to the media were isolated without breaking down media components, as indicated by the exponential portions of the graph. (F) Further analysis of measuring $\mathrm{pH}$ of the culture media subjected to different voltages and frequencies of electrical stimulation narrowed the acceptable voltage ceiling for the study. $N=3$ independent experiments, $* p<0.05$.


\section{$0.3 \mathrm{~V}$}
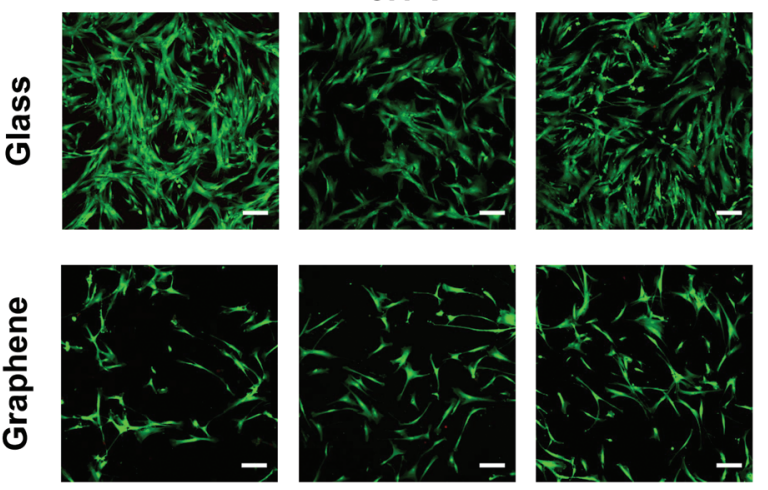

Fig. 3 Cell viability assay on glass and unpatterned graphene. A live/ dead assay using CalceinAM (live, green) and ethidium homodimer (dead, red) was conducted on glass and unpatterned graphene substrates after 24 hours of hMSC culture under the indicated stimulation voltages. All stimulation voltages were applied at $1 \mathrm{~Hz}$. For all six culture conditions, the majority of hMSCs was alive, and the cell morphology of the hMSCs cultured on unpatterned graphene was more elongated and spindle-like, especially when voltages were applied, compared to the hMSCs on glass substrates. Scale bar $=200 \mu \mathrm{m}$. were also reported several years ago by Loh and colleagues. ${ }^{24}$ It was postulated that because graphene interactions with serum proteins is limited to non-covalent, hydrophobic binding via the $\operatorname{sp}^{2} \pi-\pi$ bonds, the availability of serum protein deposits for the hMSCs to attach to are markedly decreased. ${ }^{10,11}$ In fact, they were able to quantitatively demonstrate that graphene oxide allowed for nearly three times more protein adsorption than graphene. Most importantly, the majority of hMSCs were alive across all our tested conditions further validating that the electrical stimulation setup was not cytotoxic.

\section{RUNX2 demonstrates sensitivity to electrical stimulation, but hMSCs do not commit to an osteogenic lineage}

Next, the lineage commitment of hMSCs under electrical stimulation, both on unpatterned and patterned graphene substrates, was evaluated. Of note, the substrates were not precoated with adhesive proteins and the cellular response was not biased with pre-differentiation protocols or induction media. As shown in Fig. 4, the expression of the late osteogenic marker osteopontin, a key protein involved in bone matrix mineralization, ${ }^{25-30}$ was virtually unaffected (no statistical significance detected) by any of the electrical stimulation parameters tested over 24 and 72 hours on unpatterned glass or graphene (Fig. 4A). In our previous work, we showed that 3D graphene foams promote osteogenic differentiation of hMSCs, ${ }^{31}$ and these cells were highly elongated due to the physical structure of the material; here, hMSCs on unpatterned graphene spread isotropically without confinement, which likely accounts for the observed difference. In contrast to osteopontin, the early osteogenic transcription factor RUNX2 was significantly enhanced by stimulation on all graphene substrates, regardless of patterning, and a striking enhancement of osteonpontin was observed when stimulation was coupled with the presence of physical parameters (Fig. 4B and D).

Because the late osteogenic marker osteopontin was increased only when stimulation was coupled with patterned substrates, this indicates that combined cues could enhance the conversion of hMSCs towards an osteogenic cell phenotype. Prior reports in the literature present similar results with respect to osteogenesis when hMSCs were exposed to electrical stimulus in different culture settings. Two studies using electrical current on bone marrow and adipose MSCs, using collagen gels and culture dishes, respectively, found that osteogenesis increased under high currents and voltages. ${ }^{32,33}$ Although the stimulation conditions far exceed what was used in this study, with some reports utilizing a minimum threshold current for increased osteoblastic activity at $100 \mu \mathrm{A}$ $\mathrm{cm}^{-2,34}$ it demonstrates that the combination of an appropriate material with stimulation are necessary for late stage osteogenic markers to activate osteogenic differentiation. However, upon careful inspection of Fig. 4D, osteopontin expression was modulated primarily by the presence of an electrical current. It is believed that for our cell culture system, we are able to show that osteopontin is primarily sensitive to variations in electrical current when forming bone. A novel in vitro AC stimulated cell culture system had demonstrated this exact effect when 
A)



B)

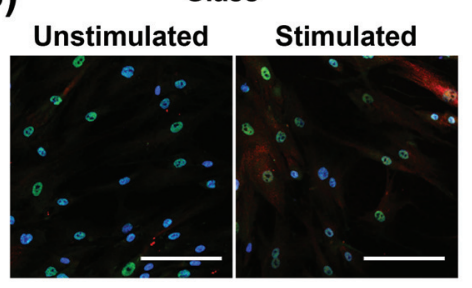

Grid Graphene



Hoechst/RUNX2/Osteopontin

D)
Flat Graphene

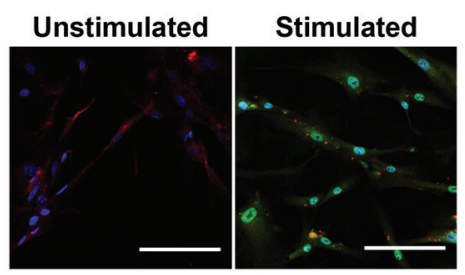

Column Graphene

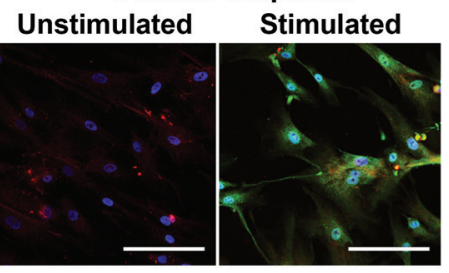

C)

RUNX2
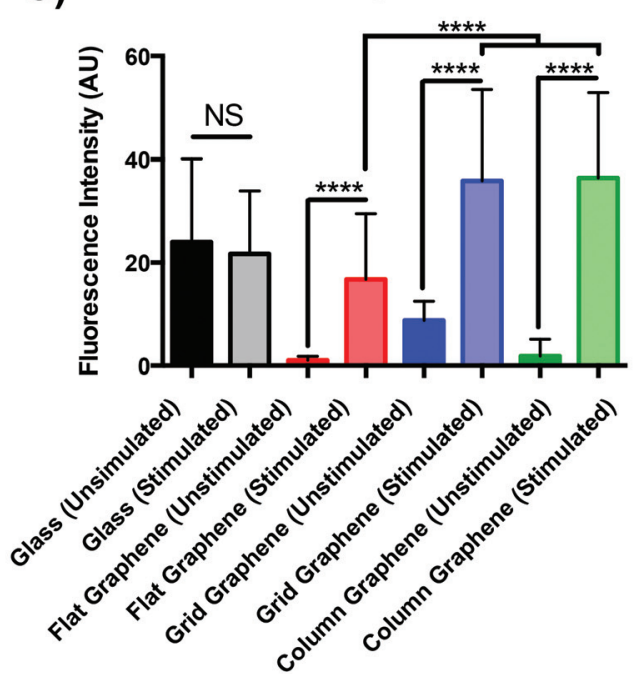
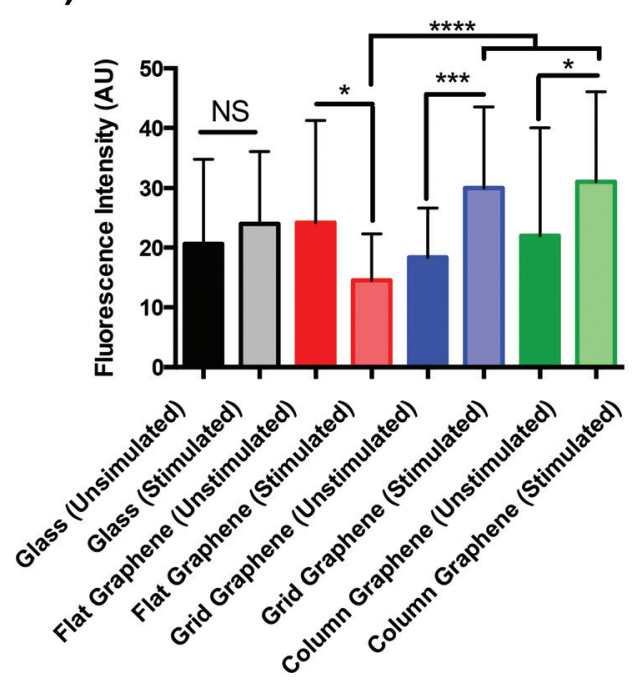

Fig. 4 Flow cytometry-based quantification of hMSCs expressing osteopontin, and immunocytochemical quantification of osteogenic markers in hMSCs grown on glass, unpatterned graphene and patterned graphene. (A) Flow cytometry-based quantification of osteopontin ${ }^{+}$cell fractions after 24 and 72 hours of stimulation reported no significant difference in the fraction of osteopontin ${ }^{+}$cells between unpatterned graphene and glass in all test conditions. (B) Representative images of hMSCs cultured on glass, unpatterned and patterned graphene, as indicated by the titles, with or without 72 hours of $0.3 \mathrm{~V}$ stimulation at $1 \mathrm{~Hz}$ (Hoechst - blue, RUNX2 - green, Osteopontin - red) (scale bar = $100 \mu \mathrm{m}$ ). Quantification of fluorescence intensity for RUNX2 (C) and osteopontin (D) is reported. All bars are mean \pm standard deviation, ${ }^{*} p<0.05,{ }^{* * *} p<0.001,{ }^{* * * *} p<0.0001$.

bone marrow hMSCs were cultured on glass or an indium tin oxide-coated glass surface. In this case, osteopontin expression was increased only in the presence of stimulation while the substrate patterns (e.g. flat) had no measurable effect. ${ }^{35}$ Additionally, osteopontin is known to carry a significant negative charge, thereby making it sensitive in carrying any bioelectrical signals. ${ }^{36}$ With respect to RUNX2, a separate study found that under increasing AC current, RUNX2 expression increased in an exponential-like pattern, which suggests this osteogenicassociated protein may have other roles in responding to external stimuli such as electrical current, thereby matching the observation we saw in our experiments. ${ }^{37}$ Finally, as our experi- mental setup had direct cell interface with graphene, the fact that we were able to observe similar responses mentioned in the aforementioned studies demonstrates the sensitivity these cell have on graphene and that only fractions of the electrical stimulation conditions are needed to elicit observable proteinlevel cell responses. While electrical stimulation could cue initial osteogenic potential on unpatterned graphene, as indicated by the alterations in RUNX2, the lack of increased late stage osteogenic protein osteopontin suggests the hMSCs have not adopted a new phenotype; however, when stimulation is combined with physical patterning, a further progression towards a more mature osteogenic phenotype is promoted. 
hMSCs undergo neurogenic differentiation on electrically stimulated, patterned graphene

Neurogenic-specific markers were also concurrently measured to investigate how the combined parameters influenced potential neurogenic differentiation of hMSCs. Expression of $\beta_{3}$-tubulin, responsible for microtubule-based neurite structure and vesicle transportation, ${ }^{38-42}$ was greatly enhanced by electrical stimulation in a voltage-dependent manner on unpatterned graphene, even at 24 hours (Fig. 5A). However, when patterned substrates were introduced, expression of $\beta_{3}$-tubulin and MAP2 were significantly enhanced in all groups tested, relative to the unpatterned counterpart, and stimulation did not further enhance the effect; MAP2, a cytoskeleton protein that binds to tubulin networks in neural cells, ${ }^{43-47}$ signal was further improved for hMSCs cultured on grid patterns coupled with electrical stimulation, but not column patterns, which agrees with previously published findings (Fig. 5B-D). Of note, stimulation on glass control substrates did not modulate any of the markers for either lineage, thereby emphasizing the role of graphene as an electrically-active, patternable culture platform.
A)

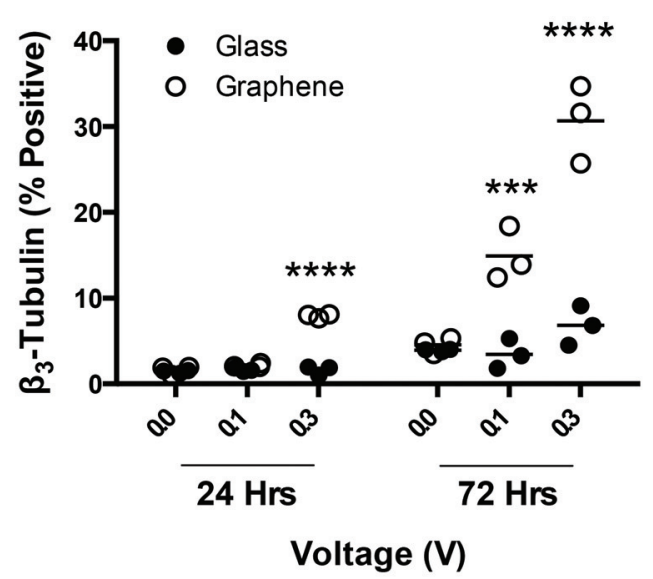

B)

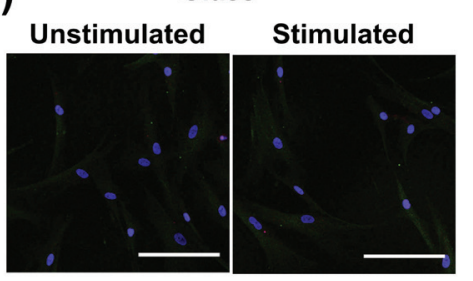

Grid Graphene

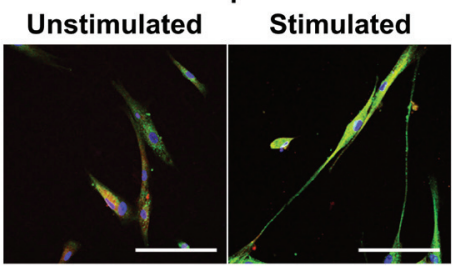

Hoechst 33258/MAP2/B3 tubulin
Flat Graphene

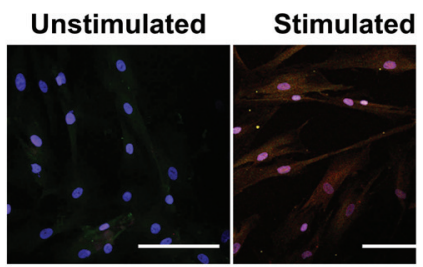

Column Graphene
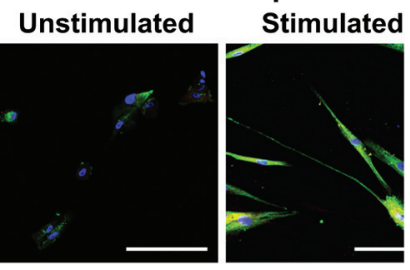

C)


D)

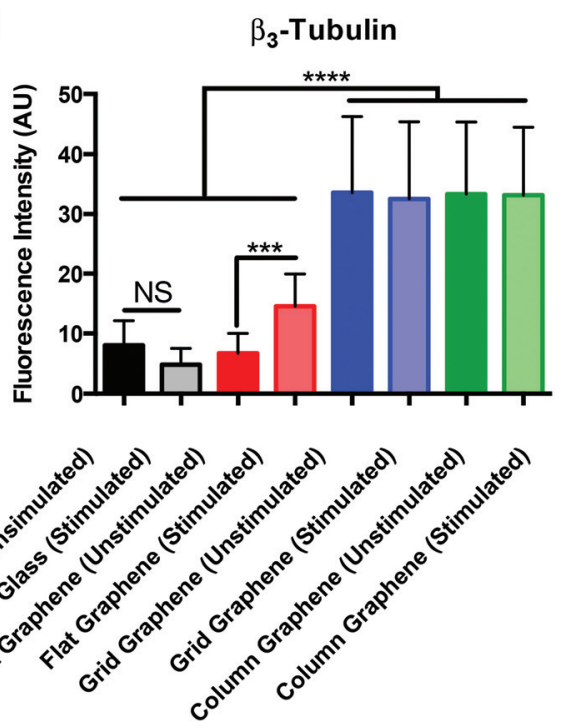

Fig. 5 Flow cytometry-based quantification of hMSCs expressing $\beta_{3}$-tubulin, and immunocytochemical quantification of neurogenic markers in hMSCs grown on glass, unpatterned graphene and patterned graphene. (A) Flow cytometry data demonstrates that when exposed to $0.3 \mathrm{~V}$, there were more positive hMSCs on graphene compared to those on glass. This difference was more pronounced after 72 hours of constant stimulation where both 0.1 and $0.3 \mathrm{~V}$ stimulation induced significantly higher expression of $\beta_{3}$-tubulin in hMSCs on graphene compared to those on the glass control. (B) Representative images of hMSCs cultured on glass, unpatterned and patterned graphene, as indicated by the titles, with or without 72 hours of $0.3 \mathrm{~V}$ stimulation at $1 \mathrm{~Hz}$ (Hoechst - blue, MAP2 - green, $\beta_{3}$-tubulin - red) (scale bar $=100 \mu \mathrm{m}$ ). Quantification of fluorescence intensity for MAP2 (C) and $\beta_{3}$-tubulin (D) is reported. All bars are mean \pm standard deviation, ${ }^{* * *} p<0.001, * * * * p<0.0001$. 
The closest publication ${ }^{12}$ that looked at neuronal differentiation in this context, which in part inspired this study, employed graphene oxide, laminin-coated substrates, and predifferentiated adipose-derived hMSCs, but these experimental conditions demonstrate critical divergences in understanding the influence of graphene on hMSC neuronal, as well as osteogenic differentiation. First, graphene oxide, as stated before, has a different surface chemistry and conductance, which would significantly affect cell response. Second, the use of laminin coating improves cell adhesion to surfaces, but can also interfere with the direct interaction between the cell and the graphene surface. ${ }^{48,49}$ Third, predifferentiating the hMSCs confounds the effect that graphene and the pattern have on the stem cells. Moreover, it has been previously shown that hMSCs can differentiate to neurons when the correct chemical supplements were added to culture media. ${ }^{50}$ Based on our experimental design where we specifically sought to minimize coupled and confounding factors, we hypothesize that the increased presence of MAP2 and $\beta_{3}$-tubulin is due to increased hMSC alignment, a typical characteristic for neurons. It has been shown in other work that substrate patterning to influence contact guidance does influence neuronal protein expression, where patterns with sharp edges and less circularity improve both MAP2 and $\beta_{3}$-tubulin expression. ${ }^{51}$ However, it should be noted that this study was not conducted under electrical stimulation and was cultured over significantly longer time intervals than we tested. Despite these differences, it is likely that the grid pattern allowed for a threshold polarization of the cells such that the additive effect of electrical stimulation could be seen. On the other hand, it is also possible that $\beta_{3}$-tubulin being a constituent part of microtubule filaments is not modulated beyond the degree of cell alignment, and that its presence is still highly dependent on proneurogenic factors (in this case, electrical stimulation) and the ability to complement with other neurogenic proteins like MAP2 to create neurite-like processes. The work presented here helps to clarify how extracellular cues modulate the lineage-specific differentiation of hMSCs in the absence of exogenous biomolecules or protein pre-coating, and highlights that combinatorial, modular nature of signals that can be presented to stem cells to more accurately control their behavior.

\section{Conclusions}

In the present study, we have demonstrated that lineage commitment of bone marrow-derived hMSCs can be controlled by modular combination of culture material type, exposure to electrical stimulation, and presentation of physical patterns. We ensured that electrical stimulation up to $0.3 \mathrm{~V}$ at $1 \mathrm{~Hz}$ did not create a cytotoxic environment through breakdown of the cell culture media or shift in media $\mathrm{pH}$ outside of the physiological range. Utilizing non-coated, pure monolayer graphene in unpatterned and patterned formats, we demonstrated a divergent response of osteogenic (RUNX2, osteopontin) and neurogenic markers $\left(\beta_{3}\right.$-tubulin, MAP2) to electrophyisical stimuli.

Yet, there remain some questions that our group desire to answer in the future to further tune the graphene patterning and the stimulation conditions during in vitro cell culture. With respect to graphene patterning, further variation in the grid and column pattern dimensions (e.g. node spacing and column widths for grid and column patterns, respectively) may elucidate how substrate topology can complement or combat the influences of electrical stimulation in our culture setup. Additionally, extending the stimulation period beyond 72 hours as well as testing intermittent changes in frequencies and voltages during an experiment could aid in optimizing neural differentiation beyond our reported findings. Because both neurogenic and osteogenic markers were observed on the patterned substrates, extended culture periods may help avoid mixed populations of pre-osteogenic cells versus more advanced neuronal differentiation. Additionally, greater numbers of cells would likely be desired by both research and clinical groups alike, hence more culture time would become a prerequisite and perhaps allowing more neuronal differentiation to occur. Regardless, these results represent a significant advance in developing a physiologically relevant culture substrate condition to direct neural differentiation of hMSCs by avoiding time consuming pre-differentiation process, laborious substrate coating, and/or expensive media-based induction.

\section{Methods}

\section{Graphene growth}

The monolayer graphene was produced on copper using chemical vapor deposition. A flat strip of $4 \mathrm{~cm}$ by $1 \mathrm{~cm}$ strip of copper was used for this growth. The growth was carried out in a standard CVD furnace (MTI OTF-1200X). Before the growth, the furnace tube was pumped down to a 0.100 mbar. The device was then annealed at 940C in $2 \mathrm{sccm} \mathrm{H}_{2}$ for 1 hours. Then, methane at $35 \mathrm{sccm}$ was introduced into the furnace for 30 minutes. Finally, the device was allowed to cooled down to $150{ }^{\circ} \mathrm{C}$ before removing the graphene.

The quality of the graphene was assessed with Raman spectroscopy. Raman spectra were recorded at ambient conditions using a Thermo Scientific DXR Raman microscope with a $100 \mu \mathrm{W}, 532 \mathrm{~nm}(\sim 2.3 \mathrm{eV})$ laser as an excitation source.

\section{Graphene transfer}

Using a Laurell WS-400B spin coater, a layer of 950, A7 Poly (methyl methacrylate) (PMMA) was spin-coated on the coppergrown graphene as a support structure for the transfer process. A heat activated adhesive was placed on the backside of the copper in order to support it during the spin coating process. The PMMA was spun coat at $4000 \mathrm{rpm}$ for 45 seconds. The sample was then heated on a hotplate at $120{ }^{\circ} \mathrm{C}$ for one minute. 
The graphene sample was then transferred onto the substrate, PDMS spun on a glass coverslip, by ferric chloride etching. The graphene/copper/PMMA stack was placed into a beaker with $\mathrm{FeCl}_{3}$ graphene side down. After 15 minutes, the copper was fully etched and the remaining PMMA/graphene structure was moved into a deionized water bath. After 10 minutes, the structure was moved again into a new deionized water bath. After 10 minutes the floating structure was lifted out of the bath onto the PDMS substrate. The water residues were then allowed to evaporate for 24 hours. This evaporation allows the graphene to adhere onto the substrate via van der Waals forces. The PMMA was subsequently dissolved in an acetone bath for 24 hours leaving a monolayer of graphene on the PDMS substrate. This PDMS substrate was made by spinning coating at $2000 \mathrm{rpm}$ a 10:1 PDMS to curing agent mixture onto a circular glass coverslip with a diameter of $1.5 \mathrm{~cm}$.

\section{Graphene patterning}

Photolithography and reactive ion etching (RIE) were used to pattern the graphene. Using a CEE 200CB spin coater, a layer of photoresist AZ5214 was spun onto the graphene sample at 4000 RPM for 45 seconds. The sample was then baked at $90{ }^{\circ} \mathrm{C}$ for 60 seconds. A chrome mask was used in order to pattern the photoresist. The sample was exposed to UV light for 8.5 seconds with hard contact using a Karl Suss MA-6 mask aligner. The sample was placed in a developer bath, AZ 351B at 1: 4 dilution with DI water, for 60 seconds. Utilizing a Trion Technologies Minilock II RIE machine, the sample underwent oxygen plasma etching for 15 seconds at $120 \mathrm{~W}$ with $30 \mathrm{sccm}$ of $\mathrm{O}_{2}$ in order to apply the photoresist pattern onto the graphene. The photoresist was then completely removed by soaking in an acetone bath for 60 minutes.

\section{Electrostimulation setup}

First, $100 \mu \mathrm{l}$ of $\mathrm{H} 20 \mathrm{E}$ silver-based conductive epoxy was placed onto the graphene substrate, with the exception for the column pattern graphene substrate where a line of the epoxy mixture was placed near the edge of the sample. A meter-long insulated platinum wire was then placed into the epoxy and baked at $200{ }^{\circ} \mathrm{C}$ for $10-15$ minutes. The samples were placed into a 24 well plate. Another meter-long insulated wire was stripped of its insulation at the end and covered in $\mathrm{H} 20 \mathrm{E}$ epoxy. The wire was cured with the same process stated earlier. This wire acted as the counter electrode in the cell media. The counter electrodes were added to each sample well and super glued down at the top so that the glue does not contact the media. This final setup is depicted in Fig. 1. The cell pacer device was composed of an Arduino UNO and a voltage divider circuit. The cell stimulation circuit was connected to the sample wells by alligator clips.

\section{Cyclic voltammetry of cell culture media}

A cyclic voltammetry experiment was conducted on the cell culture media in order to estimate the voltage range where minimal electrochemical reactions (local pH change) would occur. The experiment was run from $-1 \mathrm{~V}$ to $+1 \mathrm{~V}$ for ten cycles.

\section{Preliminary electrostimulation and $\mathrm{pH}$ experiment}

The samples were stimulated without cells at three voltages: $0.500 \mathrm{~V}, 0.300 \mathrm{~V}$, and $0.100 \mathrm{~V}$. These voltages were chosen based on data from the cyclic voltammetry experiment. Each voltage group was tested at three different frequencies with 1 microsecond pulses: $1 \mathrm{~Hz}, 3 \mathrm{~Hz}$, and $5 \mathrm{~Hz}$. Each experimental group was conducted in duplicate so that 18 samples were used. The $\mathrm{pH}$ was measured after the samples sat in the incubator for an hour. The samples were incubated for an hour with the elevated concentration of $\mathrm{CO}_{2}$ in the incubator to reach equilibrium with the $\mathrm{CO}_{2}$ concentration of the cell media. After 24 hours of stimulation, the $\mathrm{pH}$ was measured again. The well plate was sealed with parafilm during transport in order to prevent the lower $\mathrm{CO}_{2}$ levels of the air from causing diffusion of $\mathrm{CO}_{2}$ out of the cell media.

\section{Cell culture}

In order to prevent cells from adhering to the PDMS, the samples are soaked in a Pluronics ${ }^{\odot}$ bath. The bath was composed of 10 grams of Pluronic ${ }^{\circledR}$ and $100 \mathrm{~mL}$ of PBS. The samples were left in the solution for 2 hours. The samples were then rinsed in PBS and UV sterilized for 30 minutes on each side. Human bone marrow-derived mesenchymal stem cells (hMSCs) were purchased from Lonza (Walkersville, MD) and used for cell experiments at passage 5. hMSCs were cultured in complete media composed of alpha-minimum essential media with nucleosides (Life Technologies, Carlsbad, CA), 16.7\% heat-inactivated fetal bovine serum (Life Technologies), $1 \%$ penicillin/streptomycin (Life Technologies), and $4 \mu \mathrm{g} \mathrm{ml} \mathrm{m}^{-1}$ plasmocin prophylactic agent (InvivoGen, San Diego, CA) in a humidified incubator at $37{ }^{\circ} \mathrm{C}$ and $5 \% \mathrm{CO}_{2}$. Media was replaced every 3 days. hMSCs were detached from tissue culture flasks at $\sim 80 \%$ confluence with $0.05 \%$ trypsin-EDTA and passaged at $100-500$ cells per $\mathrm{cm}^{2}$ For all cell experiments, hMSCs were seeded on substrates at a density of 10000 viable cells per $\mathrm{cm}^{2}$, as determined by Trypan Blue, and cultured for specified time periods in each experiment. A silicon ring with silicon grease was used to hold the substrates down and the cell solution in place.

\section{Live/dead assay}

The cells were allowed to attach for 24 hours. The cell media was then removed. 1:1000 dilution of CalceinAM and Ethidium Homodimer (Life Technologies) was pipetted into PBS to create the live/dead solution, and $400 \mu \mathrm{l}$ of this mixture was placed in each well for 15 minutes. The live/dead solution was replaced with $400 \mu \mathrm{L}$ of fresh cell media, and the cells were imaged. Representative images are selected from $N=3$ experimental replicates.

\section{Flow cytometry}

hMSCs were cultured on test graphene substrates for 24 and 72 hours in the absence or presence of electrical stimulation 
and then detached with Trypsin (Invitrogen, Carlsbad, CA). After adding complete media to deactivate the trypsin, cells were collected by centrifugation. Cells were then washed with FACS buffer (PBS without $\mathrm{Ca} / \mathrm{Mg}^{2+}, 1 \% \mathrm{FBS}, 0.1 \% \mathrm{NaN}_{3}$ ) twice and fixed in $1 \%$ paraformaldehyde at room temperature for 10 minutes. The cells were then permeabilized using FACS buffer with $0.1 \%$ saponin; washed once in FACS buffer; and then centrifuged to a pellet. The supernatant was discarded. Class III $\beta 3$ tubulin (MAB1637, EMD Millipore, Billerica, MA) and Osteopontin (AB8448, Abcam, Cambridge, MA) were diluted 1:100 in FACS buffer plus $0.1 \%$ Triton-X 100 and treated on the cells ( $50 \mu$ per sample). The cells were incubated with primary antibodies for 1 hour at $4{ }^{\circ} \mathrm{C}$; washed once in $1 \mathrm{ml}$ FACS buffer plus $0.1 \%$ Triton-X 100; and centrifuged; and the supernatant was discarded. Secondary antibodies specific to the primary IgG isotype were diluted in FACS buffer plus $0.1 \%$ Triton-X 100 with a final sample volume of $100 \mu \mathrm{l}$ at $1: 1000$ dilution. The cells were incubated for 60 minutes in the dark at $4{ }^{\circ} \mathrm{C}$; washed in FACS buffer plus $0.1 \%$ Triton-X 100; and resuspended in $300 \mu \mathrm{l}$ FACS buffer for analysis. Results were analyzed using FlowJo v8.5.2 (FlowJo LLC, Ashland, OR).

\section{Immunostaining}

hMSCs cultured on graphene or glass were fixed in $4 \%$ paraformaldehyde for 15 minutes at room temperature and permeabilized in $0.1 \%$ Triton-X 100 for 1 hour at room temperature. Samples were blocked with Odyssey Blocking Buffer (LI-Cor Biosciences, Lincoln, NE) and incubated for 1 hour at room temperature. MAP2 (AB5622, EMD Millipore), Class III $\beta_{3}$-tubulin (MAB1637, EMD Millipore), Osteopontin (AB8448, Abcam), and Runx2 (AB76956, Abcam) were added in Odyssey Blocking Buffer (1:100 dilution) and incubated overnight at $4{ }^{\circ} \mathrm{C}$. After multiple washing with $0.1 \%$ Tween 20 in PBS and 1× PBS twice, Alexa Fluor 488 (Invitrogen) and Alexa Fluor 562 (Invitrogen) were diluted $(1: 10000)$ in the same solution as the primary antibodies and incubated at room temperature for 1 hour. The samples were washed with $0.1 \%$ Tween 20 in PBS twice and $1 \times$ PBS twice and treated with Gold Antifade Reagent with DAPI (Invitrogen). Slides were examined under a Zeiss LSM 710 confocal microscope (Carl Zeiss, Oberkochen, Germany) with a preset gain for all images taken. Image analysis was performed using ImageJ (National Institutes of Health, Bethesda, MD, USA). Briefly, composite fluorescence images were split into their respective channels and converted to gray scale. Whole cells were selected as the region of interest and gray signal was quantified. To remove potential background fluorescence, three cell-void areas were measured for gray signal intensity, averaged, and this value was subtracted from all gray values reported for selected cells. Representative images are selected from $N=3$ experimental replicates.

\section{Statistical analysis}

Graphpad Prism (Graphpad Software, Inc, La Jolla, CA) was used for statistical analysis of the results from the flow cyto- metry experiment. Two-way ANOVA was conducted for the flow cytometry experiments ( $N=3$ experimental replicates) with Sidak's post-hoc test. One-way ANOVA was conducted for the image-based experiments quantifying stain intensity $(N=3$ independent experiments with $n=50$ cells for each culture condition taken across the three independent experiments) with Tukey's post-hoc test.

\section{Acknowledgements}

This research was funded and supported by NSF CBET BME 1056046, NSF DMR BMAT 1506717, NIH EB 019509 and AHA GRNT25890018. This study was also financially supported by the Faculty Research Assistance Program of Yonsei University College of Medicine for 2016 (6-2016-0031). DAB was partially supported by the Vanderbilt Medical Scientist Training Program T32 training grant GM007347. This study also used resources provided by the Vanderbilt Institute of Nanoscale Sciences and Engineering (VINSE), a facility renovated under NSF ARI-R2 DMR-0963361.

\section{References}

1 A. Kumar and J. P. Brockes, Trends Neurosci., 2012, 35, 691699.

2 H. L. Nye, J. A. Cameron, E. A. Chernoff and D. L. Stocum, Dev. Dyn., 2003, 226, 280-294.

3 Y. Rinkevich, D. T. Montoro, E. Muhonen, G. G. Walmsley, D. Lo, M. Hasegawa, M. Januszyk, A. J. Connolly, I. L. Weissman and M. T. Longaker, Proc. Natl. Acad. Sci. U. S. A., 2014, 111, 9846-9851.

4 H. T. Nguyen, C. Wei, J. K. Chow, L. Nguy, H. K. Nguyen and C. E. Schmidt, J. Neural. Eng., 2013, 10, 046011.

5 S. L. Bowers, I. Banerjee and T. A. Baudino, J. Mol. Cell Cardiol., 2010, 48, 474-482.

6 C. S. Barros, S. J. Franco and U. Muller, Cold Spring Harbor Perspect. Biol., 2011, 3, a005108.

7 J. C. Kwok, G. Dick, D. Wang and J. W. Fawcett, Dev. Neurobiol., 2011, 71, 1073-1089.

8 A. F. Paulson, M. S. Prasad, A. H. Thuringer and P. Manzerra, Cell Adhes. Migr., 2014, 8, 19-28.

9 Y. Li, M. Wu, D. Zhao, Z. Wei, W. Zhong, X. Wang, Z. Liang and Z. Li, Sci. Rep., 2015, 5, 17817.

10 A. K. Geim and K. S. Novoselov, Nat. Mater., 2007, 6, 183191.

11 K. S. Novoselov, A. K. Geim, S. V. Morozov, D. Jiang, Y. Zhang, S. V. Dubonos, I. V. Grigorieva and A. A. Firsov, Science, 2004, 306, 666-669.

12 T. H. Kim, S. Shah, L. Yang, P. T. Yin, M. K. Hossain, B. Conley, J. W. Choi and K. B. Lee, ACS Nano, 2015, 9, 3780-3790.

13 X. Li, W. Cai, J. An, S. Kim, J. Nah, D. Yang, R. Piner, A. Velamakanni, I. Jung, E. Tutuc, S. K. Banerjee, L. Colombo and R. S. Ruoff, Science, 2009, 324, 1312-1314. 
14 S. Stankovich, D. A. Dikin, R. D. Piner, K. A. Kohlhaas, A. Kleinhammes, Y. Jia, Y. Wu, S. T. Nguyen and R. S. Ruoff, Carbon, 2007, 45, 1558-1565.

15 A. C. Ferrari, Solid State Commun., 2007, 143, 47-57.

16 J. L. Tan, W. Liu, C. M. Nelson, S. Raghavan and C. S. Chen, Tissue Eng., 2004, 10, 865-872.

17 M. R. Cho, IEEE Trans. Plasma Sci., 2002, 30, 1504-1515.

18 R. K. Aaron and D. M. Ciombor, J. Orthop. Res., 1996, 14, 582-589.

19 F. A. Armstrong, H. A. O. Hill, B. N. Oliver and N. J. Walton, J. Am. Chem. Soc., 1984, 106, 921-923.

20 J. S. Park, H. N. Yang, D. G. Woo, S. Y. Jeon, H. J. Do, S. H. Huh, N. H. Kim, J. H. Kim and K. H. Park, Biomaterials, 2012, 33, 7300-7308.

21 K. Abbasnia, A. Ghanbari, M. Abedian, A. Ghanbari, S. Sharififar and H. Azari, Anat. Cell Biol., 2015, 48, 104113.

22 M. A. Matos and M. T. Cicerone, Biotechnol. Prog., 2010, 26, 664-670.

23 C. L. Ross, M. Siriwardane, G. Almeida-Porada, C. D. Porada, P. Brink, G. J. Christ and B. S. Harrison, Stem Cell Res., 2015, 15, 96-108.

24 W. C. Lee, C. H. Lim, H. Shi, L. A. Tang, Y. Wang, C. T. Lim and K. P. Loh, ACS Nano, 2011, 5, 7334-7341.

25 M. D. McKee, W. N. Addison and M. T. Kaartinen, Cells Tissues Organs, 2005, 181, 176-188.

26 G. K. Hunter, J. O’Young, B. Grohe, M. Karttunen and H. A. Goldberg, Langmuir, 2010, 26, 18639-18646.

27 D. T. Denhardt and X. Guo, FASEB J., 1993, 7, 1475-1482.

28 Q. Chen, P. Shou, L. Zhang, C. Xu, C. Zheng, Y. Han, W. Li, Y. Huang, X. Zhang, C. Shao, A. I. Roberts, A. B. Rabson, G. Ren, Y. Zhang, Y. Wang, D. T. Denhardt and Y. Shi, Stem Cells, 2014, 32, 327-337.

29 S. P. Bruder, D. J. Fink and A. I. Caplan, J. Cell. Biochem., 1994, 56, 283-294.

30 W. N. Addison, D. L. Masica, J. J. Gray and M. D. McKee, J. Bone Miner. Res., 2010, 25, 695-705.

31 S. W. Crowder, D. Prasai, R. Rath, D. A. Balikov, H. Bae, K. I. Bolotin and H. J. Sung, Nanoscale, 2013, 5, 4171-4176.
32 C. M. Creecy, C. F. O’Neill, B. P. Arulanandam, V. L. Sylvia, C. S. Navara and R. Bizios, Tissue Eng., Part A, 2013, 19, 467-474.

33 K. E. Hammerick, A. W. James, Z. Huang, F. B. Prinz and M. T. Longaker, Tissue Eng., Part A, 2010, 16, 917-931.

34 Q. Wang, S. Zhong, J. Ouyang, L. Jiang, Z. Zhang, Y. Xie and S. Luo, Clin. Orthop. Relat. Res, 1998, 259-268.

35 M. E. Wechsler, B. P. Hermann and R. Bizios, Tissue Eng., Part C, Methods, 2016, 22, 155-164.

36 K. X. Wang and D. T. Denhardt, Cytokine Growth Factor Rev., 2008, 19, 333-345.

37 J. A. Rey Cubillos, L. Lareo, S. Gutiérrez and M. Godoy Corredor, Rev. Med., 2012, 20, 14-20.

38 S. A. Lewis, M. G. Lee and N. J. Cowan, J. Cell Biol., 1985, 101, 852-861.

39 S. P. Memberg and A. K. Hall, J. Neurobiol., 1995, 27, 26-43.

40 A. J. Roskams, X. Cai and G. V. Ronnett, Neuroscience, 1998, 83, 191-200.

41 S. S. Han, D. Y. Kang, T. Mujtaba, M. S. Rao and I. Fischer, Exp. Neurol., 2002, 177, 360-375.

42 O. von Bohlen Und Halbach, Cell Tissue Res., 2007, 329, 409-420.

43 J. G. Izant and J. R. McIntosh, Proc. Natl. Acad. Sci. U. S. A., 1980, 77, 4741-4745.

44 G. Huber and A. Matus, J. Neurosci., 1984, 4, 151-160.

45 E. E. Geisert Jr., H. G. Johnson and L. I. Binder, Proc. Natl. Acad. Sci. U. S. A., 1990, 87, 3967-3971.

46 L. Dehmelt and S. Halpain, Genome Biol., 2005, 6, 204.

47 J. H. Dinsmore and F. Solomon, Cell, 1991, 64, 817-826.

48 S. L. Goodman, R. Deutzmann and K. von der Mark, J. Cell Biol., 1987, 105, 589-598.

49 M. Manthorpe, E. Engvall, E. Ruoslahti, F. M. Longo, G. E. Davis and S. Varon, J. Cell Biol., 1983, 97, 1882-1890.

50 J. Sanchez-Ramos, S. Song, F. Cardozo-Pelaez, C. Hazzi, T. Stedeford, A. Willing, T. B. Freeman, S. Saporta, W. Janssen, N. Patel, D. R. Cooper and P. R. Sanberg, Exp. Neurol., 2000, 164, 247-256.

51 J. Lee, A. A. Abdeen and K. A. Kilian, Sci. Rep., 2014, 4, 5188. 